\title{
THE HARTMAN-WATSON DISTRIBUTION REVISITED: ASYMPTOTICS FOR PRICING ASIAN OPTIONS
}

\author{
STEFAN GERHOLD, ${ }^{*}$ Vienna University of Technology
}

\begin{abstract}
Barrieu, Rouault and Yor (2004) determined asymptotics for the logarithm of the distribution function of the Hartman-Watson distribution. We determine the asymptotics of the density. This refinement can be applied to the pricing of Asian options in the Black-Scholes model.
\end{abstract}

Keywords: Hartman-Watson distribution; Asian option; saddle point method

2010 Mathematics Subject Classification: Primary 62E20

Secondary $60 \mathrm{~J} 65$

\section{Introduction and main result}

The distribution of the integral of geometric Brownian motion has attracted a lot of interest, in particular because it is needed to calculate the price of Asian options in the Black-Scholes model. Yor [16] obtained the formula

$$
\mathrm{P}\left[A_{t}^{(v)} \in \mathrm{d} u \mid W_{t}+v t=x\right]=\frac{\sqrt{2 \pi t}}{u} \exp \left(\frac{x^{2}}{2 t}-\frac{1+\mathrm{e}^{2 x}}{2 u}\right) I_{0}\left(\frac{\mathrm{e}^{x}}{u}\right) f_{\mathrm{e}^{x} / u}(t) \mathrm{d} u,
$$

where $I_{v}$ denotes, as usual, the modified Bessel function of the first kind, and

$$
A_{t}^{(v)}=\int_{0}^{t} \exp \left(2\left(W_{h}+v h\right)\right) \mathrm{d} h
$$

where $W$ is a standard Brownian motion. The present note focuses on the function $f_{r}(t)$ in (1), which is the density of the Hartman-Watson distribution [7], [15]. It is defined for a positive parameter $r$ by the Laplace transform

$$
\int_{0}^{\infty} \mathrm{e}^{-u t} f_{r}(t) \mathrm{d} t=\frac{I_{\sqrt{2 u}}(r)}{I_{0}(r)}, \quad \operatorname{Re}(u)>0 .
$$

Small-time asymptotics of the conditional density (1) correspond to left-tail asymptotics of $f_{r}(t)$. Numerical problems in the evaluation of (1) for small $t$ prompted Barrieu et al. [1] to analyze the left tail of the Hartman-Watson distribution asymptotically. Using the Gärtner-Ellis theorem from large deviations theory, they obtained the asymptotics

$$
F_{r}(t)=\exp \left(-\frac{\log (1 / t)^{2}}{2 t}+o\left(\frac{\log (1 / t)^{2}}{t}\right)\right) \quad \text { as } t \rightarrow 0
$$

Received 25 November 2010; revision received 25 February 2011.

* Postal address: Vienna University of Technology, Wiedner Hauptstraße 8-10, A-1040 Vienna, Austria.

Email address: sgerhold@fam.tuwien.ac.at 
for the distribution function. However, this result is not immediately applicable to the calculation of (1). Barrieu et al. [1] wrote that 'the standard asymptotic methods (e.g. the saddle point method) do not seem to be suitable for this study', and that they 'are not able to refine these results for the Hartman-Watson density itself'.

In fact, the saddle point method [2, Chapter 4], [14, Section II.4] is applicable to the Laplace inversion integral

$$
f_{r}(t)=\frac{1}{2 \mathrm{i} \pi} \int_{-\mathrm{i} \infty}^{+\mathrm{i} \infty} \mathrm{e}^{u t} \frac{I_{\sqrt{2 u}}(r)}{I_{0}(r)} \mathrm{d} u
$$

for the density $f_{r}(t)$, but needs some care. First, replacing $I_{\sqrt{2 u}}(r)$ by an asymptotic approximation relieves us from studying potentially difficult monotonicity properties of the modified Bessel function, and allows us to formulate the result in a way that avoids roots of equations involving the Bessel function. Second, it turns out that elementary approximations of the integrand's saddle point lead to integration contours that are too far away from the saddle to make the method work. An approach based on a contour through the exact saddle point establishes the following asymptotics for the density $f_{r}(t)$. For brevity, we write

$$
\rho=\log \frac{r}{2 \sqrt{2}}
$$

Theorem 1. For $t>0$, denote by $u_{0}(t)$ the largest solution of the equation

$$
t=\frac{\log u}{2 \sqrt{2 u}}-\frac{\rho}{\sqrt{2 u}}+\frac{1}{4 u}
$$

which exists for all sufficiently small $t$. Then the Hartman-Watson density satisfies

$$
\begin{aligned}
f_{r}(t) & =\frac{\sqrt{\mathrm{e}}}{\pi I_{0}(r)} \sqrt{\frac{u_{0}(t)}{\log u_{0}(t)-2-2 \rho}} \mathrm{e}^{-t u_{0}(t)+\sqrt{2 u_{0}(t)}}\left(1+O\left(\sqrt{t} \log \left(\frac{1}{t}\right)^{2}\right)\right) \\
& =\frac{\sqrt{\mathrm{e}}}{2 \pi I_{0}(r)} \frac{\log (1 / t)^{1 / 2}}{t} \mathrm{e}^{-t u_{0}(t)+\sqrt{2 u_{0}(t)}}\left(1+O\left(\frac{\log \log (1 / t)}{\log (1 / t)}\right)\right)
\end{aligned}
$$

as $t \rightarrow 0$.

Formula (4) gives a much better approximation than (5); the simplification in (5) is of little use, since $u_{0}(t)$ has to be computed anyway to evaluate (4) or (5) numerically.

To obtain a feel for the growth of the exponential in (4), we expand $u_{0}(t)$ by bootstrapping (cf. [2, Section 2.4]):

$$
u_{0}(t)=\frac{\log (1 / t)^{2}}{2 t^{2}}\left(1+\frac{2 \log \log (1 / t)}{\log (1 / t)}-\frac{2 \rho+\log 2}{\log (1 / t)}+o\left(\frac{1}{\log (1 / t)}\right)\right) .
$$

Therefore, the exponent in (4) has the expansion

$$
\begin{aligned}
-t u_{0}(t)+\sqrt{2 u_{0}(t)}= & -\frac{\log (1 / t)^{2}}{2 t}-\frac{\log (1 / t) \log \log (1 / t)}{t} \\
& +\left(1+\rho+\frac{1}{2} \log 2\right) \frac{\log (1 / t)}{t}+o\left(\frac{\log (1 / t)}{t}\right) .
\end{aligned}
$$


This shows in particular that the formula

$$
f_{r}(t)=\exp \left(-\frac{\log (1 / t)^{2}}{2 t}+o\left(\frac{\log (1 / t)^{2}}{t}\right)\right)
$$

obtained from (2) by formal differentiation, is correct.

For numerical accuracy, it is certainly preferable to use (4) as it is, without replacing the exponent by (7); still, expansion (6) can serve as a good initial guess when computing the root of (3). In this way, the leading term of $f_{r}(t)$ can be calculated effortlessly even for extremely small values of $t$, say $t=10^{-50}$.

\section{Analysis of the Laplace inversion integral}

The Laplace inversion formula yields the representation

$$
f_{r}(t)=\frac{1}{2 \mathrm{i} \pi} \int_{R-\mathrm{i} \infty}^{R+\mathrm{i} \infty} \mathrm{e}^{u t} \frac{I_{\sqrt{2 u}}(r)}{I_{0}(r)} \mathrm{d} u
$$

where $R>0$, so that the integration contour lies in the right half-plane. To estimate the growth of $f_{r}(t)$ near $t=0$, we have to investigate the singularity at $\infty$ of the integrand. For a large index, the modified Bessel function admits the expansion [8], [13, p. 225]

$$
I_{v}(r) \sim\left(\frac{r}{2}\right)^{v} \mathrm{e}^{v} v^{-v-1 / 2}\left(c_{0}+\frac{c_{1}}{v}+\cdots\right),
$$

where the $c_{i}$ are constants, with $c_{0}=1 / \sqrt{2 \pi}$. This holds for $v \rightarrow \infty$, uniformly with respect to $\arg (v)$, as long as $\arg (v)$ is bounded away from $\pm \pi$. Horn [8] showed (9) for $J_{v}(r)$, the Bessel function of the first kind, but from the relation $I_{v}(r)=\mathrm{e}^{-v \pi \mathrm{i} / 2} J_{v}\left(r \mathrm{e}^{\pi \mathrm{i} / 2}\right)$, one easily sees that replacing $J_{v}(r)$ by $I_{v}(r)$ only affects the constants $c_{1}, c_{2}, \ldots$ in this expansion. If we let the real part $R$ of our integration contour tend to $\infty$ as $t \rightarrow 0$, we therefore have

$$
\begin{aligned}
f_{r}(t)= & \frac{1}{(2 \pi)^{3 / 2} \mathrm{i} I_{0}(r)} \int_{R-\mathrm{i} \infty}^{R+\mathrm{i} \infty}\left(\frac{r}{2}\right)^{\sqrt{2 u}} \mathrm{e}^{u t+\sqrt{2 u}}(\sqrt{2 u})^{-\sqrt{2 u}-1 / 2} \mathrm{~d} u\left(1+O\left(R^{-1 / 2}\right)\right) \\
= & \frac{2^{-7 / 4}}{\pi^{3 / 2} \mathrm{i} I_{0}(r)} \int_{R-\mathrm{i} \infty}^{R+\mathrm{i} \infty} \exp \left(u t-\frac{1}{2} \sqrt{2 u} \log u+\sqrt{2}(1+\rho) \sqrt{u}-\frac{1}{4} \log u\right) \mathrm{d} u \\
& \times\left(1+O\left(R^{-1 / 2}\right)\right) .
\end{aligned}
$$

The integrand of the latter integral has a saddle point, let us call it $u_{0}=u_{0}(t)$, which is found by equating the derivative to 0 . This yields (3). Shifting the integration contour through the saddle point achieves concentration, so that only a small part of the contour matters asymptotically.

In many instances of the saddle point method, it suffices to choose a contour that passes through an approximation of the saddle point. In our example, one might try to use a contour based on the first terms of (6). However, tedious calculations reveal that the concentration of the integrand around the approximate saddle point is insufficient, no matter how many terms of (6) are taken. We therefore set the real part of the integration contour to the exact saddle point, so that $R=u_{0}$ :

$$
f_{r}(t) \sim \frac{2^{-7 / 4}}{\pi^{3 / 2} \mathrm{i} I_{0}(r)} \int_{u_{0}-\mathrm{i} \infty}^{u_{0}+\mathrm{i} \infty} \exp \left(u t-\frac{1}{2} \sqrt{2 u} \log u+\sqrt{2}(1+\rho) \sqrt{u}-\frac{1}{4} \log u\right) \mathrm{d} u .
$$


Let $y$ denote the new (real) integration variable:

$$
u=u_{0}+\mathrm{i} y, \quad-\infty<y<\infty .
$$

Close to the saddle point, i.e. for small values of the new integration variable $y$, we have the uniform expansions

$$
\sqrt{u}=\sqrt{u_{0}}+\frac{\mathrm{i} y}{2 \sqrt{u_{0}}}+\frac{y^{2}}{8 u_{0}^{3 / 2}}+O\left(\frac{y^{3}}{u_{0}^{3 / 2}}\right), \quad \log u=\log u_{0}+\frac{\mathrm{i} y}{u_{0}}+\frac{y^{2}}{2 u_{0}^{2}}+O\left(\frac{y^{3}}{u_{0}^{3}}\right),
$$

and

$$
\sqrt{u} \log u=\sqrt{u_{0}} \log u_{0}+\frac{\mathrm{i} y}{\sqrt{u_{0}}}+\frac{\mathrm{i} \log \left(u_{0}\right) y}{2 \sqrt{u_{0}}}+\frac{\log \left(u_{0}\right) y^{2}}{8 u_{0}^{3 / 2}}+O\left(\frac{\log \left(u_{0}\right) y^{3}}{u_{0}^{5 / 2}}\right) .
$$

We insert these into the exponent of (10) and obtain

$$
\begin{aligned}
u t- & \frac{1}{2} \sqrt{2 u} \log u+\sqrt{2}(1+\rho) \sqrt{u}-\frac{1}{4} \log u \\
& =u_{0} t-\frac{1}{2} \sqrt{2 u_{0}} \log u_{0}+\sqrt{2}(1+\rho) \sqrt{u_{0}}-\frac{1}{4} \log u_{0}-M y^{2}+O\left(\frac{\log \left(u_{0}\right) y^{3}}{u_{0}^{5 / 2}}\right)
\end{aligned}
$$

where

$$
\begin{aligned}
M & =\frac{\sqrt{2} \log u_{0}}{16 u_{0}^{3 / 2}}-\frac{\sqrt{2}(1+\rho)}{8 u_{0}^{3 / 2}} \\
& =\frac{t^{3}}{2 \log (1 / t)^{2}}\left(1+O\left(\frac{\log \log (1 / t)}{\log (1 / t)}\right)\right) .
\end{aligned}
$$

Note that the $y$-terms in (11) vanish, because we integrate through a saddle point. We now have to identify a range

$$
-h<y<h
$$

for $y=\operatorname{Im}(u)$ that captures the main contribution to the integral (10). A good choice is

$$
h=\frac{\log (1 / t)^{2}}{t^{3 / 2}}
$$

because it satisfies $h \sqrt{M} \rightarrow \infty$, so that the integral of the local expansion (11) can be completed to a full Gaussian integral:

$$
\begin{aligned}
\int_{-h}^{h} \mathrm{e}^{-M y^{2}} \mathrm{~d} y & =\frac{1}{\sqrt{2 M}} \int_{-h \sqrt{2 M}}^{h \sqrt{2 M}} \mathrm{e}^{-w^{2} / 2} \mathrm{~d} w \\
& \sim \frac{1}{\sqrt{2 M}} \int_{-\infty}^{\infty} \mathrm{e}^{-w^{2} / 2} \mathrm{~d} w \\
& =\sqrt{\frac{\pi}{M}} \\
& \sim \frac{\sqrt{2 \pi} \log (1 / t)}{t^{3 / 2}} .
\end{aligned}
$$


Moreover, the error from (11), the local expansion at the saddle point, is $o(1)$, since

$$
\frac{\log \left(u_{0}\right) y^{3}}{u_{0}^{5 / 2}}=O\left(\sqrt{t} \log \left(\frac{1}{t}\right)^{2}\right) .
$$

We can thus determine the asymptotics of the portion $|\operatorname{Im}(u)| \leq h$ of the integral (10):

$$
\begin{aligned}
& \frac{2^{-7 / 4}}{\pi^{3 / 2} \mathrm{i} I_{0}(r)} \int_{u_{0}-\mathrm{i} h}^{u_{0}+\mathrm{i} h} \exp \left(u t-\frac{1}{2} \sqrt{2 u} \log u+\sqrt{2}(1+\rho) \sqrt{u}-\frac{1}{4} \log u\right) \mathrm{d} u \\
& \sim \frac{2^{-7 / 4}}{\pi^{3 / 2} I_{0}(r)} \exp \left(u_{0} t-\frac{1}{2} \sqrt{2 u_{0}} \log u_{0}+\sqrt{2}(1+\rho) \sqrt{u_{0}}-\frac{1}{4} \log u_{0}\right) \\
& \quad \times \int_{-h}^{h} \mathrm{e}^{-M y^{2}} \mathrm{~d} y \\
& \sim \frac{2^{-7 / 4}}{\pi I_{0}(r)} M^{-1 / 2} u_{0}^{-1 / 4} \exp \left(u_{0} t-\frac{1}{2} \sqrt{2 u_{0}} \log u_{0}+\sqrt{2}(1+\rho) \sqrt{u_{0}}\right) .
\end{aligned}
$$

This gives the right-hand side of (4), after expressing $\sqrt{u_{0}} \log u_{0}$ via the saddle point equation (3), which yields

$$
-\frac{1}{2} \sqrt{2 u_{0}} \log u_{0}=-2 u_{0} t-\rho \sqrt{2 u_{0}}+\frac{1}{2} .
$$

Furthermore, expanding $u_{0}$ by (6) gives the expression in (5). Note that we have not yet proved (4) and (5); it remains to show that the tails of (10), i.e. the parts where $|\operatorname{Im}(u)| \geq h$, are asymptotically negligible. This ensures that (16) indeed captures the asymptotics of $f_{r}(t)$.

\section{Tail estimate}

To bound the tails of (10), it suffices to consider the case $y=\operatorname{Im}(u) \geq h$, since the lower half of the tail can be handled by symmetry. We first deal with the part of the contour in (10) where the imaginary part of the integration variable is very large, say $y \geq \mathrm{e}^{\log (1 / t)^{2} / 4}$. Then $y$ clearly dominates $u_{0}$, and it follows, from

$$
\operatorname{Re}(\log u) \sim \log y, \quad \operatorname{Re}(\sqrt{u}) \sim \frac{1}{2} \sqrt{2 y}, \quad \text { and } \quad \operatorname{Re}(\sqrt{u} \log u) \sim \frac{1}{2} \sqrt{2 y} \log y,
$$

that the absolute value of the integrand is bounded by

$$
\mathrm{e}^{u_{0} t-\sqrt{y}}
$$

for small $t$. Hence, we obtain the bound

$$
\mathrm{e}^{u_{0} t} \int_{\mathrm{e}^{\log (1 / t)^{2} / 4}}^{\infty} \mathrm{e}^{-\sqrt{y}} \mathrm{~d} y \sim 2 \exp \left(u_{0} t+\frac{1}{8} \log \left(\frac{1}{t}\right)^{2}-\mathrm{e}^{\log (1 / t)^{2} / 8}\right) .
$$

Finally, we bound the portion of the integral (10) that is close to the central part, i.e.

$$
h \leq y<\mathrm{e}^{\log (1 / t)^{2} / 4} .
$$

The following lemma shows that, for small $t$, the absolute value of the integrand decreases as $y$ increases. 
Lemma 1. Let $B$ be a real number. Then, for $\operatorname{Re}(u)>0$ and sufficiently large $|u|$, the real part of $\sqrt{u} \log u+B \sqrt{u}$ decreases with respect to $|\operatorname{Im}(u)|$.

Proof. We write $u=x+\mathrm{i} y$. By symmetry, it suffices to consider the case $y>0$, so that $\arg (u)>0$. Straightforward calculations show that

$$
\frac{\partial}{\partial y} \operatorname{Re}(\sqrt{u})=\frac{1}{2}|u|^{3 / 2}\left(y \cos \frac{\arg (u)}{2}-x \sin \frac{\arg (u)}{2}\right)
$$

and

$$
\begin{aligned}
\frac{\partial}{\partial y} \operatorname{Re}(\sqrt{u} \log u)=\frac{1}{2}|u|^{3 / 2} & (\log |u|+2)\left(y \cos \frac{\arg (u)}{2}-x \sin \frac{\arg (u)}{2}\right) \\
& \left.-\arg (u)\left(x \cos \frac{\arg (u)}{2}+y \sin \frac{\arg (u)}{2}\right)\right) .
\end{aligned}
$$

Hence, we are led to investigate the sign of

$$
y\left((\log |u|+B+2)\left(\cos \frac{\arg (u)}{2}-\frac{x}{y} \sin \frac{\arg (u)}{2}\right)-\arg (u)\left(\frac{x}{y} \cos \frac{\arg (u)}{2}+\sin \frac{\arg (u)}{2}\right)\right) .
$$

Suppose that $|u|$ is so large that $\log |u|+B+2 \geq 12$. In the preceding formula, we estimate the trigonometric functions by the first term of their Taylor series at 0 , except the first cos, where we use two terms. This yields the lower bound

$$
\begin{gathered}
y\left(12\left(1-\frac{1}{8} \arg (u)^{2}-\frac{x}{2 y} \arg (u)\right)-\frac{x}{y} \arg (u)-\frac{1}{2} \arg (u)^{2}\right) \\
=y\left(12-2 \arg (u)^{2}-\frac{7 x}{y} \arg (u)\right) \\
=\left.y\left(12-2 \arctan (w)^{2}-\frac{7}{w} \arctan (w)\right)\right|_{w=y / x} .
\end{gathered}
$$

Now observe that $\arctan (w)^{2}<\pi^{2} / 4$ and $\arctan (w)<w$ for $w>0$, so that

$$
12-2 \arctan (w)^{2}-\frac{7}{w} \arctan (w)>12-\frac{\pi^{2}}{2}-7>0 .
$$

This shows that $\operatorname{Re}(\sqrt{u} \log u+B \sqrt{u})$ has a positive derivative with respect to $y$.

Therefore, we can bound part (20) of integral (10) by the value of the integrand at $y=h$ times the length of the path. By (11), (18), and

$$
\left.M y^{2}\right|_{y=h} \sim \frac{1}{2} \log \left(\frac{1}{t}\right)^{2}
$$

this amounts to a bound of the form

$$
\begin{gathered}
\exp \left(-t u_{0}(t)+\sqrt{2}(1+2 \rho) \sqrt{u_{0}(t)}-\frac{1}{2} \log \left(\frac{1}{t}\right)^{2}+o\left(\log \left(\frac{1}{t}\right)^{2}\right)\right) \mathrm{e}^{\log (1 / t)^{2} / 4} \\
=\exp \left(-t u_{0}(t)+\sqrt{2}(1+2 \rho) \sqrt{u_{0}(t)}-\frac{1}{4} \log \left(\frac{1}{t}\right)^{2}+o\left(\log \left(\frac{1}{t}\right)^{2}\right)\right)
\end{gathered}
$$


TABle 1.

\begin{tabular}{lc}
\hline \multicolumn{1}{c}{ Source of error } & Relative error \\
\hline Replace $I_{\nu}$ by (9) & $O(t / \log (1 / t))$ \\
Local expansion (see (11) and (15)) & $O\left(\sqrt{t} \log (1 / t)^{2}\right)$ \\
Gaussian tails (see (14) and (22)) & $\exp \left(-\frac{1}{2} \log (1 / t)^{2}+o\left(\log (1 / t)^{2}\right)\right)$ \\
Relative error of $M$ (see (13) and (14)) & $O(\log \log (1 / t) / \log (1 / t))$ \\
Outer tail (see (19)) & $\exp \left(-\mathrm{e}^{\log (1 / t)^{2} / 8}+o\left(\mathrm{e}^{\log (1 / t)^{2} / 8}\right)\right)$ \\
Inner tail (see (21)) & $\exp \left(-\frac{1}{4} \log (1 / t)^{2}+o\left(\log (1 / t)^{2}\right)\right)$ \\
\hline
\end{tabular}

To complete the proof of Theorem 1, let us now compare the six error terms that arose in the analysis (see Table 1). Note that the error from completing the tails of the Gaussian integral in (14) is

$$
\begin{aligned}
\sqrt{\frac{2}{M}} \int_{h \sqrt{2 M}}^{\infty} \mathrm{e}^{-w^{2} / 2} \mathrm{~d} w & \left.\sim \sqrt{\frac{2}{M}} \frac{\mathrm{e}^{-w^{2} / 2}}{w}\right|_{w=h \sqrt{2 M}} \\
& =\exp \left(-\frac{1}{2} \log \left(\frac{1}{t}\right)^{2}+o\left(\log \left(\frac{1}{t}\right)^{2}\right)\right) .
\end{aligned}
$$

If $M$ is not expanded, i.e. (12) is used, then the error from the local expansion dominates, which leads to (4). If, on the other hand, expansion (13) of $M$ is taken, then it is the relative error of $M$ that prevails.

\section{Comments}

The left tail of the Hartman-Watson distribution (see (8)) is somewhat thinner than that of the Lévy distribution (stable distribution with index $\alpha=\frac{1}{2}$ ), with density

$$
g(t)=\frac{1}{\sqrt{2 \pi t^{3}}} \mathrm{e}^{-1 / 2 t}, \quad t>0,
$$

and Laplace transform

$$
\int_{0}^{\infty} \mathrm{e}^{-u t} g(t) \mathrm{d} t=\mathrm{e}^{-\sqrt{2 u}}, \quad \operatorname{Re}(u)>0 .
$$

The faster decay of the Laplace transform of the Hartman-Watson distribution, of the order of $\exp (-\sqrt{u} \log u)$, becomes manifest in the additional factor $\log (1 / t)^{2}$ in the exponent of (8).

We now briefly comment on possible refinements of Theorem 1 . Technically speaking, continuing expansion (13) and inserting into (17) refines (5) to a full asymptotic expansion. A better expansion, respecting the asymptotic scale of the problem, can be obtained by retaining the explicit formula (12) for $M$, and taking more terms in (9) and (11). This should pose no essential difficulties; note, however, that each term in expansion (9) gives rise to a new saddle point, as the coefficient of $1 / u$ in (3) changes. Thus, the expansion will involve several implicitly defined functions of $t$ besides $u_{0}(t)$. Since the dependence of the solution of (3) on the coefficient of $1 / u$ is light, it might be possible to give an expansion that features only $u_{0}(t)$. This seems of little practical interest though. 
Concerning applications, our results can be used as a substitute for the Hartman-Watson density for small arguments, in particular, for evaluating the density of $A_{t}^{(v)}$ numerically for small $t$ (after integrating (1) with respect to the law of $W_{t}+v t$ ). The related problem of determining small-time asymptotics for the density of $A_{t}^{(v)}$ is left to future research. This density is difficult to evaluate numerically for small time [9]. Analyzing it asymptotically requires handling a double integral; see [11] and [12] for asymptotic evaluations of double integrals pertaining to other functionals of Brownian motion and the Brownian bridge, respectively. For $v=0$, the analysis should be simpler, as the density of $A_{t}^{(0)}$ can be expressed as a single integral, via Bougerol's identity [4], [10]. Note that tail asymptotics [5], [6] and large-time asymptotics [4], [10] of $A_{t}^{(v)}$ are known. See also [3] for related limit laws.

\section{References}

[1] Barrieu, P., Rouault, A. And Yor, M. (2004). A study of the Hartman-Watson distribution motivated by numerical problems related to the pricing of Asian options. J. Appl. Prob. 41, 1049-1058.

[2] De Bruijn, N. G. (1958). Asymptotic Methods in Analysis (Bibliotheca Math. 4). North-Holland, Amsterdam.

[3] Dufresne, D. (2004). The log-normal approximation in financial and other computations. Adv. Appl. Prob. 36, 747-773.

[4] Forde, M. (2011). Exact pricing and large-time asymptotics for the modified SABR model and the Brownian exponential functional. To appear in Internat. J. Theoret. Appl. Finance.

[5] Gulisashvili, A. And Stein, E. M. (2006). Asymptotic behavior of the distribution of the stock price in models with stochastic volatility: the Hull-White model. C. R. Acad. Sci. Paris 343, 519-523.

[6] Gulisashvili, A. and Stein, E. M. (2010). Asymptotic behavior of distribution densities in models with stochastic volatility. I. Math. Finance 20, 447-477.

[7] Hartman, P. and Watson, G. S. (1974). "Normal" distribution functions on spheres and the modified Bessel functions. Ann. Prob. 2, 593-607.

[8] Horn, J. (1899). Ueber lineare Differentialgleichungen mit einem veränderlichen Parameter. Math. Ann. 52, 340-362.

[9] IsHiYAma, K. (2005). Methods for evaluating density functions of exponential functionals represented as integrals of geometric Brownian motion. Methodology Comput. Appl. Prob. 7, 271-283.

[10] Matsumoto, H. and Yor, M. (2005). Exponential functionals of Brownian motion. I. Probability laws at fixed time. Prob. Surveys 2, 312-347.

[11] Tolmatz, L. (2000). Asymptotics of the distribution of the integral of the absolute value of the Brownian bridge for large arguments. Ann. Prob. 28, 132-139.

[12] Tolmatz, L. (2005). Asymptotics of the distribution of the integral of the positive part of the Brownian bridge for large arguments. J. Math. Anal. Appl. 304, 668-682.

[13] Watson, G. N. (1995). A Treatise on the Theory of Bessel Functions. Cambridge University Press.

[14] Wong, R. (1989). Asymptotic Approximations of Integrals. Academic Press, Boston, MA.

[15] Yor, M. (1980). Loi de l'indice du lacet brownien, et distribution de Hartman-Watson. Z. Wahrscheinlichkeitsth. 53, 71-95.

[16] Yor, M. (1992). On some exponential functionals of Brownian motion. Adv. Appl. Prob. 24, 509-531. 